\title{
Improved RRT_Connect Algorithm for UAV Route Planning
}

\author{
Xie $\mathrm{Ke}^{1, \mathrm{a}}$, Liu Zheng-Hua ${ }^{1,2, \mathrm{~b}}$, Wen Nuan ${ }^{1, \mathrm{a}}$ \\ ${ }^{1}$ School of Automation Science and Electrical Engineering, Beihang University, Beijing 100191, \\ China \\ ${ }^{2}$ Science and Technology on Aircraft Control Laboratory, Beihang University, Beijing 100191, \\ China \\ aemail: xieke_26@163.com, bemail:amenia@163.com
}

Key word: Route planning; RRT; UAV; Comparison method; Fuzzy Inference

\begin{abstract}
In the research of route planning, better balance between planning time and route quality should be achieved to reduce UAVs cost. This article is based on basic RRT (Rapidly-exploring Random Trees) and proposes an improved RRT_Connect algorithm which improves the way generating new node of the tree, and changes the parameters by the fuzzy inference according to the circumstance. Simulation and experiments prove that the improved RRT_Connect algorithm improves the efficiency of the planning and optimizes the route.
\end{abstract}

\section{Introduction}

UAV( Unmanned Aerial Vehicle) plays an important role for its tiny bulk, low cost and strong viability. The route planning of UAVs is finding out a route from initial position to goal position while some performance of it can be optimal or suboptimal.

Route planning algorithms include $\mathrm{A}^{*}$ algorithm, artificial potential field, genetic algorithm. All of these need the model of the space and obstacles and the complexity of the calculation grows exponentially with the expansion of the search space. RRT is an effective route planning algorithm proposed by La Valle in $1998^{[1]}$. The main advantage of it is that it needs no model of the search space and it can find out the feasible solution rapidly while its complexity of calculation won't grow with the expansion of the search space. The shortage of it is the feasible solution may not be the optimal one.

Because of these features of RRT, some improved RRT algorithms are put forward. Aiming at the directional problem in RRT's growth, other algorithms are combined with RRT, like artificial potential field ${ }^{[2]}$ and direction factor is introduced to it. As for the problem that route planned may not be the optimal one, inspire information ${ }^{[3][4]}$ is used and the route can be optimized by repeating the algorithm and choosing the best one ${ }^{[5]}$.Some hybrid algorithms optimize parameters of RRT ${ }^{[9 \sim 10]}$ to adapt to the circumstance and solve other problems while it is growing ${ }^{[6 \sim 7]}$.

This article proposes a new improved RRT_Connect algorithm that can optimize the way generating nodes while growth. Fuzzy inference is used to adjust the parameters. This algorithm can improve the efficiency of the planning and optimize the route.

\section{Basic RRT Algorithm}

RRT is an effective route planning algorithm in multiple dimension space. It needs no model of the search space and can search the whole space rapidly. It takes the initial position as the root node and increases leaf nodes by sampling randomly to expand the tree. When the goal position becomes one of leaf nodes or the distance between them is smaller than a threshold, an effective route can be figured out in this tree. A brief description of basic RRT algorithm is listed below:

$T$ : The random tree to be extended;

$Q$ : Node;

$S_{\text {free }}$ : The total areas of the search space without obstacles; 
$\varepsilon: \quad$ Extension step;

$\operatorname{Area}(Q)$ : The area in which distance to $Q$ smaller than a threshold;

(1)Take initial node $Q_{\text {init }}$ as root node of $T$, and add it in the tree;

(2)Generate $X_{\text {rand }}$ randomly in $S_{\text {free }}$;

(3)Find out $Q_{\text {near }}$, which is the nearest node to $Q_{\text {rand }}$ of $T$;

(4)Generate temporary node $Q_{\text {temp }}$ by extending a step form $Q_{\text {near }}$ to $Q_{\text {rand }}$;

(5)If the route between $Q_{\text {near }}$ and $Q_{\text {temp }}$ doesn't collide with any obstacles, $Q_{\text {new }}=Q_{\text {temp }}$, otherwise $Q_{\text {temp }}$ is abandoned;

(6)Repeat step (2) (5) until $Q_{\text {new }}=Q_{\text {goal }}$ or $Q_{\text {new }} \in \operatorname{Area}\left(Q_{\text {goal }}\right)$;

(7)Get the effective route between $Q_{\text {init }}$ and $Q_{\text {goal }}$ in the tree;

\section{Goal-directed RRT}

Though basic RRT can spread the nodes all over the search space, it grows without any guidance about the growing direction. If it grows to a direction which is opposite to the goal, many extra nodes would be generated which reduces the efficiency of planning. To change this situation, the RRT algorithm used mostly now is goal-directed RRT, which means there is some probability that RRT can grow to the goal.

A random probability $P_{g}$ is used in goal-directed RRT. Before get $Q_{\text {rand }}$ generate a random decimal number $P$ between 0 and 1 and compare it to $P_{g}$,if $P<P_{g}$, the tree extends to random direction, otherwise it extends to the goal, which means $Q_{\text {rand }}=Q_{\text {goal }}$. In this way, RRT can get directional growth to the goal, which improving the search efficiency and fasten the rate.

\section{Improved RRT_Connect}

Goal-directed RRT may make the tree sink into the corner if it explores the obstacles in real time and there are no definite rules to set the parameters. Aiming at these problems, an improved RRT_Connect algorithm is proposed here.

\section{RRT_Connect based on cost function}

RRT_Connect is to set up two RRT, one of which starts at the initial node while the other at the goal node. They are guided by the last node of the other tree while growing and the way to grow is same to basic RRT. RRT_Connect can explore the circumstance of both the initial position and goal position and reduce extra search. While distance of nearest nodes of one tree to the other is smaller than a threshold, connect the two trees and find out the route reversely.

In RRT_Connect ,the two trees grow guided by the last node of the other tree, which will cause the direct the tree grow to may deviate largely to the extension node and make the node not the optimal one. To get a better extension node while grow to the goal, cost function is used here to guide the tree's growth.

Record the route cost $h\left(\mathrm{q}_{t}\right)$ while the trees grow. The value of $h\left(\mathrm{q}_{t}\right)$ is the product of the number of nodes from start node to current node and the length of extending step $\varepsilon$. While $P>P_{g}$,calculating the value of cost function $\varphi\left(q_{t}\right)$ of nodes of the other tree

$$
\phi\left(q_{t}\right)=f\left(q_{t}\right)+h\left(q_{t}\right)
$$

While $f\left(q_{t}\right)$ is the heuristic information whose value is the distance from this node to goal node of the current tree. To simply the calculation, diagonal distance is used here

$$
f\left(q_{t}\right)=\max \left(\left|q_{e}(x)-q_{t}(x)\right|,\left|q_{e}(y)-q_{t}(y)\right|\right)+(\sqrt{2}-1) \min \left(\left|q_{e}(x)-q_{t}(x)\right|,\left|q_{e}(y)-q_{t}(y)\right|\right)
$$


$q_{e}$ is the last node of current tree, whose coordinate is $\left(q_{e}(x), q_{e}(y)\right), q_{t}$ is node of the other tree.

Choose the node of the other tree whose value of the cost function is smallest to be the guide node and the heuristic information can guide the tree grows to a direction which leads to a better route.

\section{Comparison Method}

In basic RRT algorithm, when $P<P_{g}$, the tree extends randomly from $Q_{\text {near }}$ to $Q_{\text {rand }}$, which is lack of the guidance of known information and leads to the difference of routes planned. To optimize the extension of the tree and get an approximate solution, here proposes comparison method to get leaf nodes when extending randomly:

(1)Generate $Q_{\text {rand }}$ in search space;

(2)Find out $k$ nodes, $\left(Q_{\text {near } 1}, Q_{\text {near } 2}, \ldots, Q_{\text {neark }}\right)$, in current tree which are nearest to $Q_{\text {rand }}$;

(3)Extend a step respectively from these nodes to $Q_{\text {rand }}$, and get $k$ temporary nodes, $\left(Q_{\text {temp } 1}, Q_{\text {temp } 2}, \ldots, Q_{\text {tempk }}\right)$, then calculate their value of cost function $\phi\left(q_{i}\right)$

$$
\phi\left(q_{i}\right)=g\left(q_{i}\right)+h\left(q_{i}\right)
$$

In which $g\left(q_{i}\right)$ is the Euclidean distance from temporary node $Q_{\text {tempi }}$ to $Q_{\text {rand }}$

$$
g\left(q_{i}\right)=\sqrt{\left[Q_{\text {rand }}(\mathrm{x})-Q_{\text {tempi }}(x)\right]^{2}+\left[Q_{\text {rand }}(y)-Q_{\text {tempi }}(y)\right]^{2}}
$$

And $h\left(q_{i}\right)$ is route cost from the initial node of the tree to $Q_{\text {tempi }}$, whose value can be calculated with the method in 3.1.2.

(4)Choose the node which has smallest $\phi\left(q_{i}\right)$ to be the extension node;

With comparison method, the algorithm can filtrate the nodes of the tree and make the growth of the whole tree steady, direct and rapid, and optimizes the route planned.

\section{RRT_Connect based on fuzzy inference}

Random probability $P_{g}$ and extension step $\varepsilon$ is very important in basic RRT. Whether the tree emphasizes the goal-directed extension or random extension depends on $P_{g}$. If $P_{g}$ is small, the tree is partial to extend to the goal, while it may weaken the ability to avoid collision and sink into corners, especially in the situation that the goal is surrounded obstacles. If $P_{g}$ is large, it will strengthen the ability to search the whole space and rapidly get an effective route, while it cannot approach the goal rapidly for lack of directional guidance. The most time consuming part in RRT is calculation and comparison of all nodes to find out $Q_{\text {near }}$ in the tree while extending to $Q_{\text {rand }}$. Therefore, excessive extension will cause time wasting. If $\varepsilon$ is large, the tree cannot implement effective avoidance, especially in the circumstance that obtains many obstacles which are closely distributed. If $\varepsilon$ is small, the times of extension will increase which will cause overmuch search and extension while there exists little obstacles. In a conclusion, the value of $P_{g}$ and $\varepsilon$ have strong dependency upon the circumstance, therefore, different $P_{g}$ and $\varepsilon$ in different circumstance is necessary and will improve search efficiency, avoid unnecessary extension.

To solve this problem, fuzzy inference based on current circumstance is used to get appropriate $P_{g}$ and $\varepsilon$.In simple obstacle environment, $P_{g}$ should be small while $\varepsilon$ should be large to strengthen the directivity. In complex obstacle environment, $P_{g}$ should be large while $\varepsilon$ should be small to improve the ability of searching the space to find out effective route and avoid collision.

Firstly, initial the value of $P_{g 1} 、 P_{g 2} 、 \varepsilon_{1} 、 \varepsilon_{2}$, which are random probability and extension step of the two trees. $P_{g 1} 、 P_{g 2}$ are set to be 0.5 while the value of $\varepsilon_{1} 、 \varepsilon_{2}$ depends on the size of the space. The value of these parameters will be updated by the fuzzy inference based on the obstacles in current 
environment.

Take $\mathrm{N}$ as the numbers of obstacles in current circumstance to all obstacles ratio and $\mathrm{L}$ as smallest distance of obstacles and current node to extension step ratio, de is extension step to length of an average step. Devise the domain of variables and fuzzy division number as follows:

$\mathrm{N} \in[0,0.15,0.3], \mathrm{T}(\mathrm{N}) \in\{\mathrm{PS}, \mathrm{PM}, \mathrm{PB}\}$;

$\mathrm{L} \in[0,0.5,1,1.5,2], \mathrm{T}(\mathrm{N}) \in\{\mathrm{PS}, \mathrm{PM}, \mathrm{PB}\}$;

$P_{g} \in[0.2,0.4,0.8], \mathrm{T}(\mathrm{N}) \in\{\mathrm{PS}, \mathrm{PM}, \mathrm{PB}\}$;

de $\in[0.5,0.76,1,1.25,1.5], T(N) \in\{P S, P M, P B\}$;

PS, PM, PB present respectively “Positive Small”,” Positive Medium”,” Positive Big”.

Table. 1. Fuzzy rules of Pg

\begin{tabular}{|c|c|c|c|}
\hline $\mathrm{L} N$ & PS & PM & PB \\
\hline PS & PM & PS & PB \\
\hline PM & PS & PM & PB \\
\hline PB & PS & PB & PM \\
\hline
\end{tabular}

Table.2. Fuzzy rules of de

Table.3. Membership of $\mathrm{N}$

\begin{tabular}{|c|c|c|c|c|c|}
\hline N & 0 & 0.05 & 0.15 & 0.25 & 0.3 \\
\hline PS & 1 & 0.6 & 0 & 0 & 0 \\
\hline PM & 0 & 0.3 & 1 & 0.3 & 0 \\
\hline PB & 0 & 0 & 0 & 0.6 & 1 \\
\hline
\end{tabular}

\begin{tabular}{|c|c|c|c|}
\hline $\mathrm{L} N$ & PS & PM & PB \\
\hline PS & PM & PS & PS \\
\hline PM & PB & PM & PS \\
\hline PB & PB & PB & PM \\
\hline
\end{tabular}

Table.4. Membership of L

\begin{tabular}{|c|c|c|c|c|c|}
\hline $\mathrm{T}(\mathrm{L})^{\mathrm{L}}$ & 0 & 0.5 & 1 & 1.5 & 2 \\
\hline PS & 1 & 0.5 & 0 & 0 & 0 \\
\hline $\mathrm{PM}$ & 0 & 0.5 & 1 & 0.5 & 0 \\
\hline PB & 0 & 0 & 0 & 0.5 & 1 \\
\hline
\end{tabular}

Table.5. Membership of Pg

\begin{tabular}{|c|c|c|c|}
\hline Pg & 0.2 & 0.4 & 0.8 \\
T(Pg) & & & \\
\hline PS & 1 & 0.5 & 0 \\
\hline PM & 0 & 1 & 0 \\
\hline PB & 0 & 0.5 & 1 \\
\hline
\end{tabular}

Table.6. Membership of de

\begin{tabular}{|c|c|c|c|c|c|}
\hline $\begin{array}{r}\text { de } \\
\text { T(de) }\end{array}$ & 0.5 & 0.75 & 1 & 1.25 & 1.5 \\
\hline PS & 1 & 0.3 & 0 & 0 & 0 \\
\hline PM & 0 & 0.7 & 1 & 0.7 & 0 \\
\hline PB & 0 & 0 & 0 & 0.3 & 1 \\
\hline
\end{tabular}

\section{Steps of improved RRT_Connect}

Take one of the two RRT as example, the flow chart of improved RRT_Connect is showed below: 


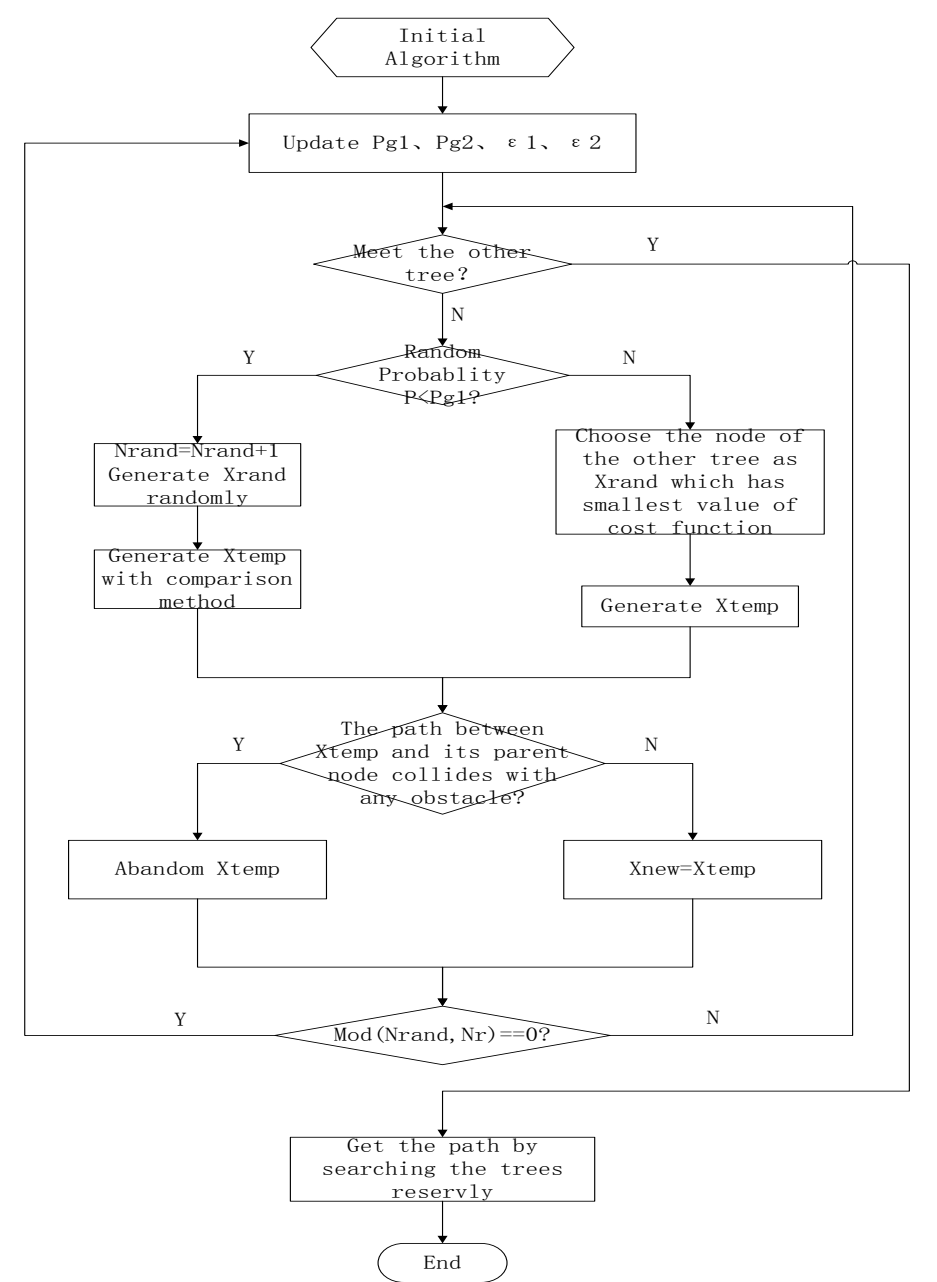

Fig. 1. The flow chart of improved RRT_Connect

\section{The result of simulation experiments and analysis}

The results of simulation of improved RRT_Connect algorithm are showed below:

1) Size: $1000 \times 1000$,Number of obstacles: 100

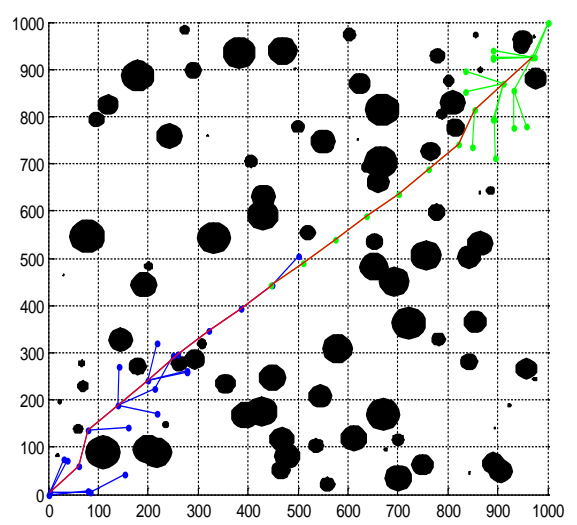

(a)Growth chart

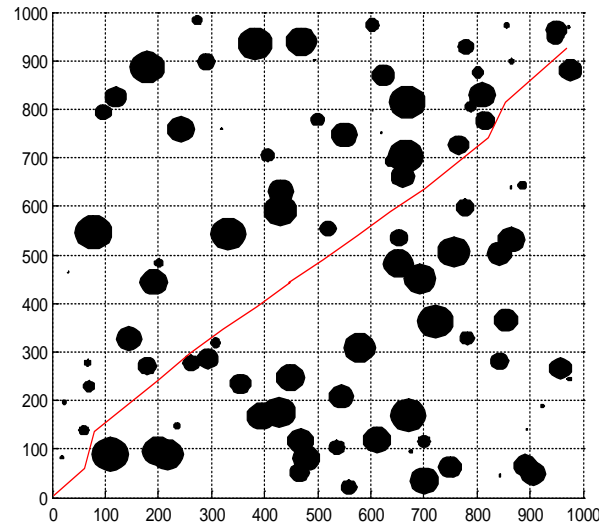

(b) Route chart

Fig.2. Simulation results

Table.7. Length of route and times of random extension

\begin{tabular}{|l|c|c|}
\hline & Length of route & Nrand \\
\hline RRT_Connect based on cost function & 1709.2 & \\
\hline RRT_Connect with comparison method & 1538.5 & 172 \\
\hline RRT_Connct with fuzzy inference & 1447.4 & 78 \\
\hline
\end{tabular}



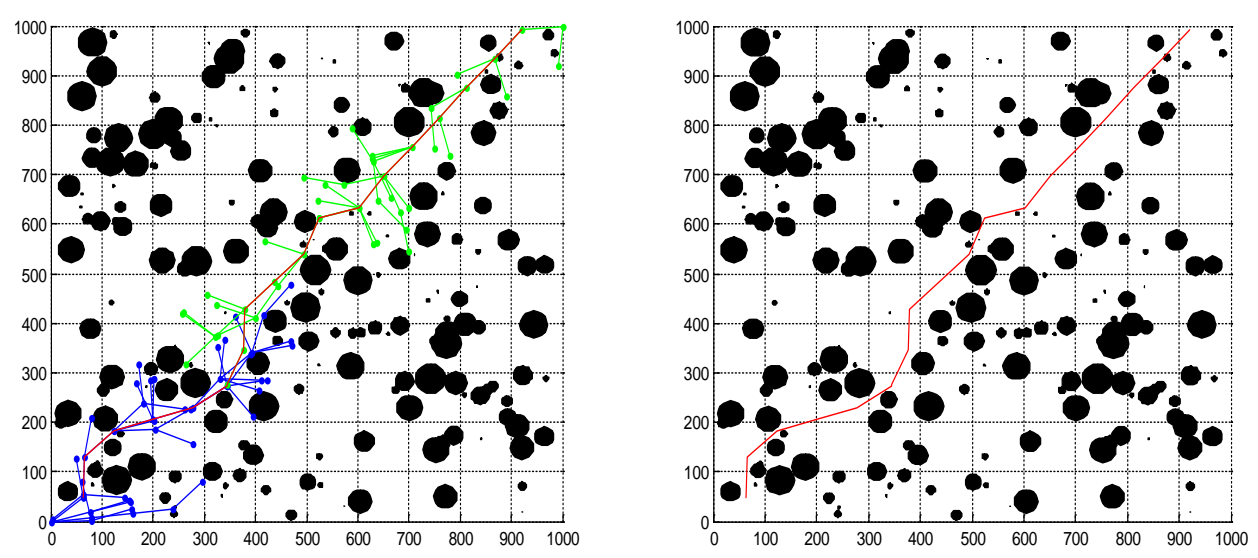

Size:1000×1000,Numbers of obstacles:200

(a) Growth chart

Fig.3. Simulation results

(b) Route chart

Table.8. Length of route and times of random extension

\begin{tabular}{|l|c|c|}
\hline & Length of route & Nrand \\
\hline RRT_Connect based on cost function & 1769.5 & \\
\hline RRT_Connect with comparison method & 1593.6 & 367 \\
\hline RRT_Connct with fuzzy inference & 1521.5 & 162 \\
\hline
\end{tabular}

As showed in figures and tables above, RRT_Connect with comparison method optimizes the route compared to RRT_Connect based on cost function. Route planned by RRT_Connect with fuzzy inference is smoother and the times of random extension are smaller. RRT_Connect with fuzzy inference can get effective parameters as the circumstance changes.

\section{Conclusion}

This article proposes an improved RRT_Connect algorithm. Basic RRT algorithm cannot balance the randomness and directivity of RRT while growing and has no definite rules to set the parameters, and the route get by basic RRT each time differs much and may not be the optimal one. Aiming at problems listed above, the improved RRT_Connect algorithm can solve them by optimizing the way RRT_Connect generates leaf nodes and changing value of parameters in its growth based on fuzzy inference, which fulfill the optimizing of the route and reduce extra nodes to increase the planning efficiency. The simulation experiments prove the algorithm's effectiveness and validity.

\section{Acknowledgement}

This work was partly supported by the grants of National Natural Science Foundation of China (No. 61305132) and Aeronautical Science Foundation of China (No. 20135751040).

\section{Reference:}

[1] Steven M.LaValle. Rapidly-exploring random trees: a new tool for route planning[R]. Dept. Lowa State University of Computer Science, 1998.

[2] He L. Based on Improved RRT with Artificial Potential Field Hybrid

Algorithm of Robot Soccer Route Planning Research [D]. Xi an University of Science and Technology, 2011.In Chinese

[3] Li J, Liu S, Zhang B, et al. RRT-A* Motion planning algorithm for non-holonomic mobile robot[C]. //SICE Annual Conference (SICE), 2014 Proceedings of the. IEEE, 2014:1833 - 1838.In Chinese 
[4] Wang B, Jin M, Xie Z, et al. RRT Algorithm Based On Heuristic [J]. Machine Manufacturing, 2007, 45(12):1-4. DOI:10.3969/j.issn.1000-4998.2007.12.001. In Chinese

[5] FENG Lin, JIA Jinghui.Improved algorithm of RRT route planning based on comparison optimization.Computer Engineering and Applications, 2011, 47 (3) : 210-213.DOI:10.3778/ j.issn.1002-8331.2011.03.062. In Chinese

[6] Runarsson T P, Yao X. X.: Search biases in constrained evolutionary optimization[J]. IEEE Transactions on Systems, Man, and Cybernetics Part C-Applications and Reviews, 2005, 35:233--243.

[7] Takahama T, Sakai S. Constrained optimization by applying the /spl alpha/ constrained method to the nonlinear simplex method with mutations.[J]. Evolutionary Computation, IEEE Transactions on, 2005, (5):437-45

[8] Qin F, Pan G,Yang J, Improved RRT Algorithm of UAV Route Planning[J].MIRCOELECTRONIC\&COMPUTER, 2012, 29(2).In Chinese

[9] Wang F,Feng $\mathrm{N}, \mathrm{Hu} \mathrm{X}$, An Improved Route Planning Algorithm Based On RRT-ConCon[J],Journal of Dalian University of Technology, 2014, (6):637-643.DOI:10.7511/dllgxb201406007.In Chinese

[10] Liu H, Rao K, Xiao F. Obstacle guided RRT route planner with region classification for changing environments[C]. //Robotics and Biomimetics (ROBIO), 2013 IEEE International Conference on. IEEE, 2013:164 - 171. 